\title{
Artigos Originais \\ Perfil Sócio-demográfico e Clínico de uma Amostra de Fumantes Portugueses: Implicações do Sexo para a Intervenção na Cessação Tabágica
}

\author{
Eleonora C. V. Costa ${ }^{1}$ \\ Portuguese Catholic University \\ Maria Júlia Valério \\ Ivone Pascoal \\ Centro Hospitalar de Vila Nova de Gaia Portugal \\ Ana Margarida Trovisqueira \\ Portuguese Catholic University
}

\begin{abstract}
RESUMO - As medidas de cessação tabágica mostram resultados positivos na diminuição da morbidade e mortalidade associadas ao consumo do tabaco. Esses programas possuem maior eficácia quando se adequam às especificidades dos tabagistas. Este estudo teve como objetivo analisar as diferenças de sexo em uma amostra de 100 fumantes portugueses, através da administração de um questionário sócio-demográfico e clínico. Observaram-se diferenças de sexo no número de cigarros consumidos por dia, no momento do dia e no contexto social de maior consumo, no residir com fumantes, na pressão social para deixar de fumar e no sono. As implicações clínicas dos resultados foram discutidas no sentido de otimizar a prestação dos serviços de saúde em contexto de cessação tabágica.
\end{abstract}

Palavras-chave: tabagismo, diferenças sexuais (humano), cessação tabágica, promoção da saúde

\section{Sociodemographic and Clinical Profile of a Smoking Portuguese Sample: Sex Implications for Intervention in Smoking Cessation}

\begin{abstract}
Smoking cessation programs reduce the morbidity and mortality associated with tobacco consumption. Such programs are more effective when they are tailored to the specific characteristics of smokers. In this study we analyze sex differences within a group of 100 portuguese smokers, by administering a sociodemographic and clinical questionnaire. The results show sex differences in the number of cigarettes smoked per day, in the time of day and the social contexts connected with greater consumption, in co-habitation with other smokers, in response to social pressures to quit smoking, and in incidence of sleep problems. We discuss the implications of the results for the optimization of the smoking cessation programs offered in healthcare settings.
\end{abstract}

Keywords: smoking, human sex differences, smoking cessation program, health promotion

O tabagismo permanece um dos principais fatores de risco para morbidade e morte prematura. O uso do tabaco surge associado a várias doenças e continua a ser causa de morte em mais de 100 milhões de pessoas em todo o mundo, sobretudo nos países com rendimento médio-baixo (World Health Organization [WHO], 2012).

A prevalência do tabagismo na Europa está estimada em $28,6 \%$ sendo mais elevada nos homens $(40 \%)$ do que nas mulheres $(18,2 \%)$ (WHO, 2009), embora esses dados variem em função da região europeia. Na população portuguesa, os resultados de um estudo epidemiológico realizado com uma amostra de 35229 indivíduos apontam para 20,9\% de fumantes, sendo a prevalência nos homens de $31 \%$ e de $11,8 \%$ nas mulheres (Machado, Nicolau, \& Dias, 2009). Diferentes estudos realizados em Portugal confirmam que a prevalência do tabagismo é mais elevada nos homens do que nas mulheres (Campos, 2008; Direção Geral da Saúde, 2013; Trigo; 2007).

Tratando-se o tabagismo de um fator de risco modificável, a cessação tabágica constitui uma das principais estratégias de prevenção das doenças associadas ao consumo do tabaco. Nesse sentido, vários países têm adotado medidas de controle

1 Endereço para correspondência: Universidade Católica Portuguesa, Praça da Faculdade, Braga, Portugal . CEP: 1.4710.297. E-mail: eleonora@braga.ucp.pt do tabaco eficazes, com boa relação custo-benefício. A cessação dos hábitos tabágicos aumenta a expectativa de vida em qualquer idade (apesar de o aumento ser superior na população mais jovem). Um estudo realizado com exfumantes revelou que parar de fumar aos 40 anos aumenta a expectativa de vida em cerca de 4,6 anos, tal como o número de anos livres de doença aumenta para três anos (Streppel, Boshuizen, Ocké, Kok, \& Kromhout, 2007). A cessação tabágica mostra-se de fato uma medida eficaz na promoção de um melhor prognóstico de doentes com os mais diversos problemas de saúde (e.g., redução do risco de cataratas; Ratneswaran et al., 2014), na promoção dos comportamentos saudáveis em pacientes psiquiátricos internados (Solty, Crockford, White, \& Currie, 2009), na diminuição do risco em pacientes com doenças respiratórias (Tonnesen et al., 2007), na redução do consumo em mulheres grávidas com hábitos tabágicos (Pullon et al., 2003), na melhoria significativa da função pulmonar (Chaudhuri et al., 2006; Willemse et al., 2005; Willemse, Postma, Timens, \& Hacken, 2004) e na melhoria dos resultados de saúde em indivíduos com HIV (Vidrine, 2009).

Contudo, a relação entre a implementação das medidas de cessação dos hábitos tabágicos e a eficácia das mesmas não é linear. Entre os preditores do sucesso dos programas 
de cessação tabágica, encontram-se, para além da eficácia da comunicação estabelecida entre o profissional de saúde e o fumante, o grau de dependência tabágica dos fumantes e suas características individuais (e.g., nível de escolaridade, história de tentativas de interrupção do consumo, estado emocional, sexo) (Nunes et al., 2007; Pereira \& Vargas, 2015; Tonnesen et al., 2007). Essas variáveis devem ser levadas em conta na construção e implementação das ações de desabituação do consumo de tabaco. De fato, as medidas de cessação tabágica apresentam maior eficácia em fumantes com escolaridade superior (e.g., elevada motivação para o abandono do consumo de cigarros é verificada em mulheres com nível educacional elevado) (Donzé, Ruffieux, \& Cornuz, 2007), fumantes com tentativas anteriores de interrupção do consumo, indivíduos não deprimidos (pacientes que evidenciam sintomatologia depressiva apresentam menos sucesso na adesão aos programas), fumantes que não possuem familiares em primeiro grau com hábitos tabágicos e fumantes do sexo masculino (Nunes et al., 2007). Essa última variável, sexo, é umas das mais referenciadas na literatura acerca da eficácia das ações de interrupção do consumo de cigarros, surgindo, em alguns estudos, o sexo feminino associado a uma menor probabilidade de sucesso (Alberg, Carter, \& Carpenter, 2007).

São várias as diferenças individuais entre homens e mulheres que podem influenciar a eficácia das medidas de cessação do consumo. Entre essas diferenças, encontra-se o receio de aumentar o peso corporal, geralmente mais elevado nas mulheres (Alberg et al., 2007; Audrain-McGovern \& Benowitz, 2011). A depressão é também apontada como uma das variáveis associadas à menor eficácia dos programas de intervenção, sendo a sua prevalência mais elevada no sexo feminino (Centers for Disease Control and Prevention, 2010). Tal como referem Pereira e Vargas (2015), o perfil predominante das mulheres em tratamento para a cessação tabágica inclui, para além de outras variáveis, o diagnóstico de depressão, sublinhando o papel da sintomatologia depressiva como fator de risco para as recaídas.

Um menor sucesso nos resultados da intervenção nos hábitos tabágicos também surge associado ao estatuto profissional do fumante. Fumantes desempregados, sobretudo do sexo masculino e com baixo status ocupacional evidenciam taxas de sucesso inferiores (Khaled, Bulloch, Exner, \& Patten, 2009).

Algumas medidas de cessação do consumo tabágico atendem às especificidades de subgrupos de fumantes (e.g., sexo; fase do ciclo de vida), contemplando necessidades próprias desses indivíduos. Essa especialização das ações interventivas exige um conhecimento científico amplo acerca dos fatores envolvidos na mudança dos hábitos tabágicos, bem como conhecimento das características da população alvo em relação a diferentes variáveis e indicadores (e.g., início do consumo, tentativas de cessação prévias, recaídas). Alguns desses programas de intervenção centram-se no Modelo Transteórico da Mudança de Proschaska e DiClemente (Prochaska, DiClemente, Velicer, Ginpi, \& Norcross, 1985), o qual contempla vários determinantes na mudança de um dado comportamento, entre os quais, o sexo. No que tange às variáveis sociocognitivas, a Teoria do Comportamento Planeado (TCP; Ajzen, 1991; Ajzen
\& Madden, 1986) tem sido aplicada à cessação tabágica e considera a intenção para deixar de fumar como um preditor, e mesmo um mediador, importante do comportamento de deixar de fumar (Afonso \& Pereira, 2013; Rise, Kovac, Kraft, \& Moan, 2008). A intenção resulta da combinação das atitudes, normas subjetivas e controle percebido. Assim, se um fumante quer deixar de fumar (atitude), a família poderá apoiar essa decisão disponibilizando-se para ajudálo (norma subjetiva). Contudo, se o fumante apresentar uma baixa perceção da sua competência para deixar de fumar (perceção de controle do comportamento), a intenção para deixar de fumar pode ser baixa apesar de as atitudes e normas subjetivas serem favoráveis. A Health Action Process Approach (HAPA) também tenta explicar a cessação tabágica e propõe uma distinção entre o processo motivacional préintencional (incluindo percepções de risco), que conduz à intenção de realizar a mudança comportamental, e o processo volitivo pós-intencional, que conduz à efetiva mudança comportamental (Schwarzer, 2008). O processo volitivo pode ser ainda subdividido em uma fase de planejamento, de ação e de manutenção. No contexto da cessação tabágica, a HAPA prediz que aqueles que não possuem a intenção de deixar de fumar (sem intenção) possuem percepções de risco mais baixas do que aqueles que possuem a intenção de cessar o tabagismo (com intenção). De fato, os estudos mostram que as percepções de risco distinguem aqueles que possuem intenção de cessar o hábito tabágico daqueles que não a possuem, sendo que aqueles que apresentam baixa motivação para deixar de fumar poderão ser beneficiados por informação sobre os sérios problemas de saúde causados pelo fumar (Williams, Herzog, \& Simmons, 2011).

De fato, conhecer as características diferenciais dos homens e das mulheres que influenciam o processo de cessação tabágica pode fornecer pistas para uma maior eficácia da intervenção. Bjornson, Connett, Lindgren, Nides e Pope (1995) sugerem que os programas de cessação tabágica devem melhorar as técnicas interventivas dirigidas às mulheres que possuem determinadas características (e.g., baixa escolaridade) e promover determinadas medidas de ação (e.g., promover o apoio social, sobretudo nas relações interpessoais mais intímas) e dedicar mais tempo de seguimento às fumantes do sexo feminino em abstinência. Rohrbaugh, Shoham e Dempsey (2009) salientaram ainda que as intervenções tabágicas devem ser dirigidas não só ao fumante mas também ao parceiro de modo a neutralizar as influências interpessoais, sobretudo quando o fumante é do sexo feminino. Adicionalmente, Christakis e Fowler (2008) avaliaram a probabilidade da diminuição do consumo de tabaco através do impacto da rede social. Os resultados mostraram que, quando um cônjuge, um irmão, um amigo ou colegas de trabalho deixam de fumar, aumenta a probabilidade de o participante também deixar de fumar. Outro estudo longitudinal, desenvolvido por Dollar, Homish, Kozlowski e Leonard (2009), mostrou que o comportamento aditivo de um membro do casal diminuía a probabilidade de cessação tabágica no cônjuge.

São vários os estudos que apontam que o sexo constitui um importante preditor da eficácia das intervenções de cessação tabágica, sobressaindo dos resultados de diferentes investigações que as mulheres apresentam mais dificuldade 
em abandonar o consumo tabágico do que os homens (Helgeson, 2016; Leventhal et al., 2007). Perkins et al. (2000) consideram que o aumento do peso corporal, as variações de humor, as alterações nas diferentes fases do ciclo menstrual e a falta de apoio social influenciam o efeito da abstinência sobretudo nas mulheres. De fato, constata-se que, uma vez iniciado o consumo por parte das mulheres, estas estão menos propensas ao abandono dos hábitos tabágicos do que os homens, evidenciando os homens, a longo prazo, maior probabilidade de cessar o consumo (Bjornson et al., 1995). Estudos mais recentes apontam que ter um parceiro consumidor é um fator determinante de recaída para as mulheres, um ano após o início da intervenção (Walsh et al., 2007; Rohrbaugh et al., 2009), tal como história de psicopatologia, nomeadamente de depressão (Centers for Disease Control and Prevention, 2010). Bjornson e colaboradores (1995) constataram que a dependência de nicotina era mais evidente para os homens e a dependência emocional para as mulheres. Nesse âmbito, uma investigação realizada em Portugal por Trigo (2007) mostrou que as mulheres fumantes portuguesas recorrem a um maior número de cigarros por dia como estratégia para reduzir o afeto negativo - dependência emocional dos cigarros.

Tratando-se o tabagismo de um comportamento de risco modificável, a adoção de medidas de cessação tabágica tem sido uma prioridade dos serviços de saúde em diferentes países. Em Portugal, as consultas de apoio aos fumantes foram desenvolvidas e são implementadas pela DirecçãoGeral da Saúde, com o grupo representativo que engloba as Administrações Regionais de Saúde e a Sociedade Portuguesa de Pneumologia, considerando o $21^{\circ}$ artigo da Lei 37/2007 (Direção Geral da Saúde, 2013). Similarmente à grande parte dos estados membros da Europa, Portugal investe na formação dos profissionais de saúde no âmbito da cessação do tabagismo, implementa a terapia de reposição de nicotina e bupropiona (WHO, 2009) e integra programas nacionais de prevenção, diagnóstico e tratamento de dependência do tabaco como parte dos cuidados de saúde primários (Direção Geral da Saúde, 2013). No entanto, e tal como em vários outros países, no nosso país, a recaída entre os fumantes que tentam cessar o consumo de tabaco é significativa.

Considerando as múltiplas variáveis envolvidas na eficácia das medidas de desabituação tabágica, bem como a elevada recidiva dos fumantes que tentam cessar o consumo tabágico, mostra-se prioritário desenvolver ações estratégicas que promovam a eficácia dos serviços de cessação tabágica, nomeadamente, atendendo às especificidades de sexo no desenvolvimento dos programas dirigidos aos fumantes portugueses. Tal como sublinha a Organização Mundial de Saúde (WHO, 2009), é importante considerar o sexo na construção de programas de promoção da saúde e educativos. Pelo exposto, foi desenhado um estudo observacional descritivo-transversal cujo principal objetivo consistiu em caracterizar, no âmbito clínico e sociodemográfico, uma amostra de pacientes portugueses em consulta de desabituação tabágica, identificando as diferenças de sexo nas variáveis estudadas.

\section{Método}

\section{Participantes}

A amostra da presente investigação foi constituída pelo universo de usuários da consulta de desabituação tabágica do Centro Hospitalar de Vila Nova de Gaia/ Espinho (CHVNG/E; Serviço de Pneumologia). Trata-se de um estudo transversal, com uma amostra de conveniência de 100 pacientes (43 mulheres e 57 homens).

Quanto às características sociodemográficas, a maioria dos participantes tem mais de 40 anos de idade (58\%), é casada $(71 \%)$ e reside com o cônjuge/companheiro e com os filhos (63\%). No âmbito profissional, 18\% dos participantes exercem funções de serviços/vendedores; $15 \%$, trabalhos não qualificados; 13\%, trabalhos técnicos; 13\% são operários; $11 \%$ possuem especialização profissional; e 3\% exercem funções em quadros superiores.

Na caraterização da amostra por sexo, verificou-se que a maioria dos participantes do sexo feminino $(72,1 \%)$ possui entre 30 e 50 anos de idade e $78,9 \%$ da amostra masculina possui entre 30 e 65 anos. A percentagem de pacientes casados é mais elevada entre os homens $(80,7 \%)$ do que entre as mulheres (58,1\%). Quanto ao estado civil, 32,6\% das pacientes são divorciadas, havendo apenas três homens na mesma condição $(5,3 \%)$. O número de participantes solteiros é mais elevado entre os homens $(n=7)$ do que entre as mulheres $(n=2)$. A maioria dos homens $(71,2 \%)$ e das mulheres $(51,2 \%)$ reside com o cônjuge e com filhos. No âmbito profissional, 32,6\% das mulheres exercem funções em quadros superiores ou possuem especialização profissional, sendo que 20,9\% das mulheres referiram ser domésticas. $\mathrm{Na}$ amostra masculina, as ocupações profissionais com percentagem mais elevada são profissões relacionadas com serviços/vendedores $(21,1 \%)$, operários $(17,5 \%)$ e trabalhos não qualificados (15,8\%). A amostra masculina mostra uma maior percentagem de participantes aposentados $(15,8 \%)$ do que a amostra feminina $(2,3 \%)$. A percentagem de desempregados é equivalente no sexo feminino $(9,3 \%)$ e no masculino $(8,8 \%)$.

\section{Procedimentos}

A amostra foi selecionada no ano de 2008, tendo sido considerados os primeiros 100 pacientes que se consultaram nesse período. $\mathrm{O}$ recrutamento se deu na consulta de desabituação tabágica do CHVNG/E (Serviço de Pneumologia) após parecer positivo da comissão de ética do referido hospital para realização desta investigação. Os pacientes foram convidados a colaborar voluntariamente no estudo no dia da consulta, sendo-lhes apresentados os objetivos do mesmo e tendo sido solicitado o preenchimento do consentimento informado. O questionário foi administrado pela psicóloga nas consultas de psicologia, individualmente a cada participante. 


\section{Instrumentos}

Neste estudo, foi utilizado o Questionário da Consulta de Desabituação Tabágica, desenvolvido por Costa e Valério (2009). Esse instrumento é constituído por 20 itens de escolha múltipla, que permitem obter dados acerca das características sociodemográficas dos participantes (idade, estado civil, profissão, agregado familiar, se vive ou trabalha com fumantes, se realiza refeições com fumantes), dos hábitos tabágicos, do contexto e dinâmica social do consumo, bem como acerca do sono do paciente. Considerando o curto período de tempo que existe para a avaliação psicológica na consulta de desabituação tabágica, foi elaborado um questionário sociodemográfico e clínico breve e não foram consideradas escalas validadas para a população portuguesa que permitiriam avaliar a gravidade de dependência e qualidade do sono. Por consequência, essa é uma limitação do estudo, pois a avaliação efetuada pelos próprios pacientes está sujeita a muitas possibilidades de erros.

\section{Análise dos Dados}

O tratamento e a análise dos dados foram efetuados através do Statistical Package for Social Sciences (IBM SPSS - versão 19). Foram utilizados procedimentos de estatística descritiva na caracterização sociodemográfica da amostra e o teste Qui-quadrado no estudo da associação entre as variáveis. No estudo das tendências das associações encontradas entre as categorias, efetuou-se a análise dos resíduos ajustados. Foi assumido como significância estatística um valor de $p<0,05$.

\section{Resultados}

Os resultados são apresentados para a amostra total, para a amostra feminina e para a amostra masculina, São evidenciadas as diferenças de sexo encontradas.

A maioria dos participantes deste estudo consome diariamente entre 10 a 20 cigarros $(49,5 \%)$, sendo que $30,3 \%$ dos pacientes consome 20 a 40 cigarros por dia e apenas $3 \%$ da amostra refere consumir menos de cinco cigarros por dia (Tabela 1). No sexo feminino, a maioria das participantes $(55,8 \%)$ consome 10 a 20 cigarros diariamente; $20,9 \%$ das pacientes consome 20 a 40 cigarros; e $18,6 \%$ referem fumar entre 5 a 10 cigarros por dia. $\mathrm{Na}$ amostra masculina, 44,6\% dos participantes consome 10 a 20 cigarros diariamente e $37,5 \% 20$ a 40 cigarros por dia. Verificou-se uma associação estatisticamente significativa entre o sexo e a quantidade de cigarros consumidos por dia $(p<0,05)$. Foi realizada uma análise dos resíduos ajustados que revelou não haver diferenças significativas entre o número de casos observados e esperados. Destaca-se que nenhuma mulher fuma diariamente mais de 40 cigarros, o que acontece com $8 \%$ dos homens.

No que concerne ao momento do dia em que se verifica um maior consumo de cigarros, constata-se que a maioria dos pacientes da amostra total $(39,6 \%)$ considera não haver um momento específico do dia em que fuma mais cigarros. Entre os demais, $27,5 \%$ dos participantes refere consumir mais cigarros durante a manhã, sendo a tarde o momento do dia associado a menor consumo (14,3\%). Verificou-se uma associação significativa entre o sexo e o momento do dia de maior consumo $(\mathrm{X} 2=10 ; \mathrm{p}=0,018)$ tendo-se procedido à análise dos resíduos ajustados. Destaca-se um maior número de mulheres $(31,7 \%)$ para as quais a noite é o momento do dia em que fumam mais, ocorrendo o inverso na amostra masculina: $32 \%$ dos homens declararam fumar mais durante a manhã.

Verifica-se também que 36,8\% dos participantes fuma mais quando está sozinho. Os que assinalam que o consumo de cigarros não difere com o fato de estar acompanhado ou sozinho totalizam $32,6 \%$ dos participantes. No sexo feminino, verifica-se que estar sozinha surge associado a um maior consumo de cigarros $(51,2 \%)$, sendo inferior a percentagem de mulheres que consume mais quando acompanhada $(34,1 \%)$. Os participantes da amostra masculina referem fumar mais quando acompanhados $(27,8 \%)$ do que sozinhos $(25,9 \%)$. A maioria dos homens considera que estar sozinho ou acompanhado não determina um maior consumo de cigarros (46.3\%). Verificou-se uma associação estatisticamente significativa entre o sexo e o contexto interpessoal de maior consumo $\left(X^{2}=11,5 ; p=0,003\right)$. A análise dos resíduos ajustados indica que existe um baixo número de homens que fuma mais quando sozinho comparativamente ao número de mulheres, bem como um elevado número de homens para os quais é indiferente estar acompanhado ou sozinho para fumar mais.

$\mathrm{Na}$ amostra total, a maioria dos pacientes não reside com fumantes $(54,5 \%)$. Na amostra feminina, é mais elevada a percentagem de mulheres que reside com fumantes $(58,1 \%)$, verificando-se o inverso na amostra masculina, em que $64,3 \%$ não residem com fumantes. Essa diferença entre o sexo feminino e o masculino revelou-se estatisticamente significativa $(p<0,05)$, conforme pode ser observado na Tabela 2.

Na tabela 3, constata-se que, na amostra total, 46,5\% dos participantes são alvo de pressão para a cessação do consumo por parte dos cônjuges, sendo os filhos a segunda principal fonte de pressão para a interrupção dos hábitos tabágicos $(21,2 \%)$. Os participantes que assinalam não sofrer qualquer pressão para deixar de fumar totalizam $8 \%$. As mulheres referem receber mais pressão dos filhos para abandonar o consumo do que do companheiro/marido, enquanto os homens assinalam maior pressão da esposa para deixar de fumar. Verificou-se uma associação estatísticamente significativa entre o sexo e a pressão exercida pelos pares para o abandono do consumo ( $\mathrm{X} 2=16,3 ; \mathrm{p}=0,003)$. Da análise dos resíduos ajustados, destaca-se que as mulheres recebem mais pressão dos seus filhos do que dos cônjuges, existindo um maior número de homens que recebem mais pressão das esposas e um menor número de homens que recebem pressão dos filhos $(71,7 \%$ dos homens recebem pressão da esposa e 30,2\% das mulheres recebem pressão do marido/ companheiro).

A tabela 3 refere-se à perceção dos participantes acerca da pressão social para cessar o consumo de cigarros. Os participantes que indicam que o efeito da pressão exercida pelos pares é positivo totalizam $42,3 \%$, sendo essa percepção mais evidente na amostra feminina $(51,6 \%)$ do que no grupo 
dos homens (37,2\%). O efeito negativo da pressão para cessar o consumo também é mais frequente nas mulheres $(38,7 \%)$ do que nos homens (20,9\%). Verificou-se uma associação estatísticamente significativa entre o sexo e a pressão exercida para o abandono do consumo $\left(X^{2}=9,46 ; p=0,024\right)$. A análise dos resíduos ajustados indicou haver mais homens para os quais o efeito da pressão exercida é variável. Inversamente, existe um menor número de mulheres para os quais o efeito da pressão é variável.

A maioria dos participantes refere sono regular sem uso da medicação (61\%), sendo a percentagem mais elevada nos homens $(71,9 \%)$ do que nas mulheres (46,5\%). Assinala-se, contudo, que a categoria "regular com medicação" possui um número reduzido de participantes $(n=6)$. As alterações no sono foram relatadas mais pelas mulheres $(41,9 \%)$ do que pelos homens $(26,3 \%)$. Verificou-se uma associação estatisticamente significativa entre a variável sexo e a classificação do sono $\left(X^{2}=8,37 ; p=0,015\right)$. A análise dos resíduos ajustados revela que um reduzido número de mulheres considera ter o sono regulado sem medicação, sendo que a maioria das pacientes apresenta o seu sono regulado pela medicação. Por sua vez, um maior número de homens apresenta um sono regulado sem medicação.

\section{Discussão}

A discussão dos resultados obtidos neste estudo encontrase organizada por área temática investigada. São discutidos, portanto, hábitos tabágicos, interação social, consumo tabágico e sono.

O número de cigarros consumidos por dia pode indicar, juntamente com outros fatores (e.g., tempo entre o acordar e o primeiro cigarro; pontuação obtida no teste de Fagerstrom; níveis de monóxido de carbono no ar expirado), o grau de dependência (Nunes et al., 2007). A quantidade de cigarros consumidos por dia constitui, assim, um importante indicador de avaliação do uso de tabaco. No presente estudo, 49,5\%

Tabela 1. Diferenças de sexo e resultados na amostra total no consumo diário de cigarros, altura do dia e contexto interpessoal de maior consumo

\begin{tabular}{|c|c|c|c|c|c|c|c|c|c|c|c|}
\hline \multicolumn{10}{|c|}{ Sexo } & \multirow{2}{*}{\multicolumn{2}{|c|}{$\begin{array}{c}\text { Amostra } \\
\text { Total }\end{array}$}} \\
\hline & \multicolumn{3}{|c|}{ Feminino $(n=43)$} & \multicolumn{3}{|c|}{ Masculino $(n=56)$} & \multirow{2}{*}{$\chi^{2}$} & \multirow{2}{*}{$g l$} & \multirow{2}{*}{$p$} & & \\
\hline & $\mathbf{n}$ & $\%$ & RA & $\mathbf{n}$ & $\%$ & $\mathbf{R A}$ & & & & $\mathbf{n}$ & $\%$ \\
\hline Quantidade de cigarros & & & & & & & 9.95 & 4 & $.04 *$ & & \\
\hline$<5$ & 2 & 4.7 & 0.8 & 1 & 1.8 & -0.8 & & & & 3 & 3 \\
\hline $5-10$ & 8 & 18.6 & 1.7 & 4 & 7.1 & -1.7 & & & & 12 & 12 \\
\hline $10-20$ & 24 & 55.8 & 1.1 & 25 & 44.6 & -1.1 & & & & 49 & 49.5 \\
\hline $20-40$ & 9 & 20.9 & 1.8 & 21 & 37.5 & -1.8 & & & & 30 & 30.3 \\
\hline$>40$ & 0 & 0 & -2 & 5 & 8.9 & 2 & & & & 5 & 5 \\
\hline Altura em que fuma mais & & & & & & & 10 & 3 & $.018 *$ & & \\
\hline Manhã & 9 & 22 & -1.1 & 16 & 32 & 1.1 & & & & 25 & 27.5 \\
\hline Tarde & 7 & 17.1 & 0.7 & 6 & 12 & -0.7 & & & & 13 & 14.3 \\
\hline Noite & 13 & 31.7 & 2.9 & 4 & 8 & -2.9 & & & & 17 & 18.7 \\
\hline Indiferente & 12 & 29.3 & -1.8 & 24 & 48 & 1.8 & & & & 36 & 39.6 \\
\hline Contexto interpessoal de maior consumo & & & & & & & 11.5 & 2 & $.003 * *$ & & \\
\hline Sozinho & 21 & 51.2 & 2.5 & 14 & 25.9 & -2.5 & & & & 35 & 36.8 \\
\hline Acompanhado & 14 & 34.1 & 0.7 & 15 & 27.8 & -0.7 & & & & 29 & 30.5 \\
\hline Indiferente & 6 & 14.6 & -3.3 & 25 & 46.3 & 3.3 & & & & 31 & 32.6 \\
\hline
\end{tabular}

Nota: $\mathrm{RA}=$ resíduos ajustados; ${ }^{*} p \leq .05 ; * * p<.01 ; * * * \leq .001$ 
dos pacientes da amostra (44,6\% dos homens e 55,8\% das mulheres) fumam entre 10 a 20 cigarros por dia. Os resultados relativos à quantidade de cigarros consumidos diariamente pela amostra masculina são equivalentes aos resultados encontrados por Trigo (2007) em um estudo realizado em Portugal. Esse autor verificou que em média os homens consumiam 17,2 cigarros por dia, comparativamente com os 12,3 cigarros por dia consumidos pelas mulheres. A margem de 12 cigarros por dia é, em média, o número de cigarros consumidos pelas mulheres mais velhas (Donzé et al., 2007), sendo compatível com a faixa etária dos 40-50 anos que caracteriza a amostra avaliada neste estudo. Porém, no estudo de Campos (2008), o número de cigarros consumidos por dia pelos homens era superior a 20, o que se mostra acima do encontrado no presente estudo. No entanto, a associação mais forte e o que distingue de fato as mulheres dos homens, é

Tabela 2. Residir com fumantes: diferenças de sexo e resultados na amostra total

\begin{tabular}{|c|c|c|c|c|c|c|c|c|c|c|c|}
\hline \multicolumn{10}{|c|}{ Sexo } & \multirow{2}{*}{\multicolumn{2}{|c|}{$\begin{array}{c}\text { Amostra } \\
\text { Total }\end{array}$}} \\
\hline & \multicolumn{3}{|c|}{ Feminino $(n=43)$} & \multicolumn{3}{|c|}{ Masculino $(n=56)$} & \multirow{2}{*}{$\chi^{2}$} & \multirow{2}{*}{$g l$} & \multirow{2}{*}{$p$} & & \\
\hline & $\mathbf{n}$ & $\%$ & RA & $\mathbf{n}$ & $\%$ & RA & & & & $\mathbf{n}$ & $\%$ \\
\hline Reside com fumantes & & & & & & & 4.93 & 1 & $.026 *$ & & \\
\hline Sim & 25 & 58.1 & - & 20 & 35.7 & - & & & & 45 & 45.5 \\
\hline Não & 18 & 41.9 & & 36 & 64.3 & & & & & 54 & 54.5 \\
\hline
\end{tabular}

Nota: $* x \leq .05 ; * * p<.01 ; * * * p \leq .001$

Tabela 3. Diferenças de sexo e resultados na amostra total na perceção da pressão social para a cessação do consumo tabágico e perceção do efeito da mesma

\begin{tabular}{|c|c|c|c|c|c|c|c|c|c|c|c|}
\hline \multicolumn{10}{|c|}{ Sexo } & \multirow{2}{*}{\multicolumn{2}{|c|}{$\begin{array}{c}\text { Amostra } \\
\text { Total }\end{array}$}} \\
\hline & \multicolumn{3}{|c|}{ Feminino $(n=43)$} & \multicolumn{3}{|c|}{ Masculino $(n=56)$} & \multirow{2}{*}{$\chi^{2}$} & \multirow{2}{*}{$g l$} & \multirow{2}{*}{$p$} & & \\
\hline & $\mathbf{n}$ & $\%$ & $\mathbf{R A}$ & $\mathbf{n}$ & $\%$ & $\mathbf{R A}$ & & & & $\mathbf{n}$ & $\%$ \\
\hline Pressão social para deixar de fumar & & & & & & & 16.3 & 4 & $.003 * *$ & & \\
\hline Não pressionado & 3 & 7 & -0.4 & 5 & 8.9 & 0.4 & & & & 8 & 8,1 \\
\hline Pressionado pelos filhos & 16 & 37.2 & 3.4 & 5 & 23.8 & -3.4 & & & & 21 & 21.2 \\
\hline Pressionado pelo cônjuge & 13 & 30.2 & -2.8 & 33 & 71.7 & 2.8 & & & & 46 & 46.5 \\
\hline Pressionado por colegas de trabalho & 2 & 4.7 & -0.3 & 0 & 0 & 0.3 & & & & 2 & 2 \\
\hline Efeito da pressão pelos pares & & & & & & & 9.46 & 3 & $.024 *$ & & \\
\hline Positivo & 16 & 51.6 & 1.2 & 16 & 37.2 & -1.2 & & & & 32 & 42.3 \\
\hline Negativo & 12 & 38.7 & 1.7 & 9 & 20.9 & -1.7 & & & & 21 & 28.4 \\
\hline Variável & 2 & 6.5 & -2.1 & 11 & 25.6 & $2-1$ & & & & 13 & 17.3 \\
\hline Indiferente & 1 & 3.2 & -1.8 & 7 & 16.3 & 1.8 & & & & 8 & 18.8 \\
\hline
\end{tabular}

Nota: $\mathrm{RA}=$ resíduos ajustados; ${ }^{*} p \leq .05 ;{ }^{* *} p<.01 ; * * * p \leq .001$

Tabela 4. Diferenças de sexo e resultados na amostra total no sono

\begin{tabular}{|c|c|c|c|c|c|c|c|c|c|c|c|}
\hline \multicolumn{10}{|c|}{ Sexo } & \multirow{2}{*}{\multicolumn{2}{|c|}{$\begin{array}{c}\text { Amostra } \\
\text { Total }\end{array}$}} \\
\hline & \multicolumn{3}{|c|}{ Feminino $(n=43)$} & \multicolumn{3}{|c|}{ Masculino $(n=56)$} & \multirow{2}{*}{$\chi^{2}$} & \multirow{2}{*}{$g l$} & \multirow{2}{*}{$p$} & & \\
\hline & $\mathbf{n}$ & $\%$ & RA & $\mathbf{n}$ & $\%$ & RA & & & & $\mathbf{n}$ & $\%$ \\
\hline Sono & & & & & & & 8.37 & 2 & $.015 *$ & & \\
\hline Regular sem medicação & 20 & 46.5 & -2.6 & 41 & 71.9 & 2.6 & & & & 61 & 61 \\
\hline Regular com medicação & 5 & 11.6 & 2.1 & 1 & 1.8 & -2.1 & & & & 6 & 6 \\
\hline Alterações do sono & 18 & 41.9 & 1.6 & 15 & 26.3 & -1.6 & & & & 33 & 33 \\
\hline
\end{tabular}


que elas não fumam 40 ou mais cigarros por dia, ao contrário dos homens $(8,5 \%)$.

Verificou-se que os homens têm tendência a fumar um maior número de cigarros por dia do que as mulheres, o que vai de encontro aos resultados de vários estudos (e.g., Campos, 2008; Direção Geral da Saúde, 2013; Trigo, 2007). Por outro lado, Rosendo, Fonseca, Guedes e Martins (2009) verificaram um menor consumo de cigarros por dia $(13,3$ cigarros/dia) no sexo feminino, em relação ao sexo masculino (22,2 cigarros/dia). A literatura psicológica refere que as diferenças de sexo no consumo de tabaco são principalmente devidas a diferentes comportamentos, tendo as suas raízes nos papéis de gênero tradicionais, nomeadamente: (a) características gerais dos papéis sexuais tradicionais conduzem à pressão social contra o tabagismo no feminino; (b) normas tradicionais dos papéis de gênero provocam diferenças nas características pessoais conduzindo a maior ou menor aceitação do fumar (por exemplo, a rebeldia entre os homens é mais aceita do que entre as mulheres e faz com que as taxas de tabagismo sejam mais elevadas nos homens); e (c) os papeis de gênero influenciam a avaliação dos custos e da utilidade de fumar (Bauer, Göhlmann, \& Sinning, 2006). Outras possibilidades de explicação relacionam-se com o tipo de dependência. Considerando que o número de cigarros é um indicador confiável do nível de dependência, poderá existir na amostra em estudo uma maior dependência de nicotina nos pacientes do sexo masculino, tal como encontrado no estudo de Bjornson e colaboradores (1995). Os homens poderão fumar uma quantidade mais elevada de cigarros de modo a obter estimulação ou para reduzir os sinais de privação da dependência. Por sua vez, o menor consumo evidenciado pela amostra feminina poderá indicar que o comportamento tabágico pode ser explicado como uma estratégia para reduzir o afeto negativo, o que poderá estar associado a uma dependência de caráter tendencialmente emocional, uma possibilidade explicativa aboradada por Trigo (2007).

Os resultados evidenciaram ainda que a amostra feminina fuma mais à noite $(31,7 \%)$, o que ocorre com apenas $8 \%$ dos pacientes do sexo masculino. Uma das possíveis hipóteses explicativas dessa variação no momento do consumo pode relacionar-se com os contextos diários distintos, assumindo a mulher a maior parte das responsabilidades domésticas e dos cuidados infantis. Em um estudo que procurou caracterizar o perfil dos indivíduos que fumam mais durante a noite, verificou-se que esse hábito estava associado a uma maior dependência e a um elevado consumo de cafeína durante o dia (Scharf, Dunbar, \& Shiffman, 2008). Analisando as implicações desses resultados, podemos destacar que os efeitos da abstinência durante a noite podem promover alterações do humor e sonolência diurna, tornando-se um fator de risco para o sucesso terapêutico. No estudo de Prosise, Bonnet, Berry e Dickel (1994), verificou-se, após abandono do consumo durante a noite, que os fumantes apresentavam despertares noturnos e demonstravam reações de irritabilidade, ansiedade e maior tensão fisica, aumentando, por sua vez, a sonolência diurna e as alterações do humor. Como indicam Scharf et al. (2008), os tratamentos devem ser revistos para as pessoas que fumam mais à noite, de modo a melhorar a qualidade do sono. Alerta-se, assim, para a possibilidade de reposição de nicotina no período noturno, enquanto estratégia terapêutica para esses fumantes.

No que concerne ao contexto interpessoal associado a maior consumo, os resultados revelaram que as mulheres fumam mais quando se encontram sozinhas $(51,2 \%)$, enquanto uma grande percentagem dos homens $(46,3 \%)$ relatam que o consumo tabágico é independente do fato de estarem acompanhados ou sozinhos. Esses resultados vão ao encontro do que tem sido revelado em outros estudos em que, na mulher, verifica-se uma representação funcional para com o cigarro, sendo este associado à fonte de prazer, alívio de sentimentos negativos, de situações de estresse, compensação da solidão, apoio em situações difíceis; enquanto que, no homem, o fenômeno grupal e a necessidade do sentimento de pertença e de identidade são as maiores influências para o tabagismo (Oliveira \& Gorayeb, 2012).

Verificou-se também neste estudo que as características dos pacientes que residem com fumantes diferem na amostra masculina e na amostra feminina. Os resultados mostraram haver um maior número de mulheres do que de homens que residem com fumantes. Perante esses resultados, coloca-se a possibilidade desse fator contextual poder interferir no sucesso dos programas de cessação tabágica ou mesmo aumentar a ocorrência de recidivas no consumo. De fato, tal como verificamos na revisão da literatura, as mulheres apresentam uma maior propensão para viver com um cônjuge fumante mostrando este ser um fator determinante nas recaídas (Rohrbaugh et al., 2009; Walsh et al., 2007), sendo as normas sociais consideradas importantes fatores envolvidos no abandono ou na manutenção do consumo. De fato, a TCP (Ajzen, 1991; Ajzen \& Madden, 1986) assume a importância das normas subjetivas na determinação da intenção de deixar de fumar, para além das atitudes e do controle percebido, pelo que, não havendo apoio na decisão de deixar de fumar nem disponibilização de ajuda, a probabilidade de mudança comportamental será menor.

Paralelamente, também os agentes de pressão social para deixar de fumar tendem a ser diferentes na amostra masculina e na feminina. Enquanto os homens percebem maior pressão por parte da esposa $(71,7 \%)$ e menor pressão exercida pelos filhos $(23,8 \%)$, as mulheres percebem maior pressão para cessar o consumo por parte dos filhos $(37,2 \%)$ e menor pressão exercida pelo cônjuge (30,2\%). A pressão social por parte dos colegas de trabalho não revelou exercer um papel importante na pressão para deixar de fumar quer nos homens quer nas mulheres. Destaca-se que apenas $2 \%$ dos pacientes da amostra total percebem pressão social para deixar de fumar por parte de amigos/colegas de trabalho, o que pode constituir um obstáculo ao sucesso dos programas interventivos, tanto nos homens como nas mulheres. Tal como apontam May e Wets (2000), o apoio social pode beneficiar a eficácia das intervenções para a cessação tabágica. De fato, esse indicador evidencia que as intervenções de cessação tabágica não se devem restringir ao contexto clínico. Para Sumner, Dunaway e Dillman (1998), os programas comunitários devem ser implementados de modo a promover a cessação tabágica e a prevenção do tabagismo junto às famílias e a outros grupos sociais. Mostra-se, desse modo, pertinente que as políticas sociais antitabagismo invistam no desenvolvimento de programas de intervenção comunitária de modo a aumentar a 
pressão social exercida nas redes sociais. De fato, o efeito da pressão para cessar o consumo tabágico foi classificado, neste estudo, sobretudo pelas mulheres $(51,6 \%)$, como positivo, apesar de não ser o indicador que prevalece ao nível das diferenças de sexo. Para os pacientes do sexo masculino, mais do que para as mulheres, o efeito da pressão social mostra-se variável podendo ser ora positivo, ora negativo, mas nunca indiferente. Mostra-se, assim, pertinente investigar os fatores que medeiam essa relação. Tal como vimos anteriormente, as pacientes do sexo feminino percebem menor pressão para a cessação do consumo por parte do marido/companheiro, tornando-se assim pertinente investigar as implicações desse fenômeno interpessoal na manutenção, na progressão e no abandono dos programas de cessação tabágica. No entanto, convém salientar a existência de dados que nos indicam que a pressão social para deixar de fumar pode não ter um efeito significativo no abandono do consumo, tal como se verificou em outro estudo com fumantes portugueses. Trigo (2007) verificou que a pressão social para deixar de fumar exercida por familiares ou cônjuges não tem sido apontada como razão para deixar de fumar, o que mostra que os fumantes não parecem ser regidos por um locus de controle externo. Outros autores também revelam que a pressão social tem mais importância para iniciar o consumo, sobretudo na adolescência, e menos na cessação tabágica no adulto (e.g., Oliveira \& Gorayeb, 2012). No entanto, e apesar desses dados, os resultados obtidos na amostra feminina do presente estudo indicam um efeito positivo da pressão social, permanecendo, no entanto, desconhecidos os atributos que sustentam essa avaliação subjectiva. Em suma, a pressão social para deixar de fumar pode favorecer a intenção comportamental, pelo apoio e disponibilização da família em ajudar na implementação do comportamento, tal como preconizado pela TCP (Ajzen, 1991; Ajzen \& Madden, 1986). Contudo, não basta que as normas subjetivas sejam favoráveis, sendo necessário desenvolver atitudes favoráveis e percepção de controle do comportamento por parte do fumante (Afonso \& Pereira, 2013; Rise et al., 2008).

Por fim, dos resultados obtidos neste estudo, destacase o reduzido número de mulheres fumantes com o sono regulado sem medicação, observando-se que a maioria das pacientes recorre a fármacos para conciliar o sono. Verificase o oposto no sexo masculino, apresentando um maior número de homens um sono regulado sem medicação. As mulheres fumam mais à noite e apresentam o sono regulado com medicação e os homens não apresentam alterações de sono, pelo que podemos supor que as mulheres, mais do que os homens, evidenciam alterações na qualidade do sono. De fato, a literatura indica que o tabagismo está associado a uma pior qualidade do sono (Catalán, Gallego-Gómez, GonzálezMoro, \& Simonelli-Muñoz, 2014). Por exemplo, o tabaco é considerado um fator de risco moderado para desenvolver bruxismo do sono (Macedo, 2008). Adicionalmente, a diminuição de 50\% no consumo da nicotina pode desencadear sintomas de abstinência nos indivíduos dependentes, que incluem, para além da ansiedade, irritabilidade, aumento do apetite, alterações cognitivas, os distúrbios do sono (insônia e sonolência diurna), entre outros (Balbani \& Montovani, 2005). De sublinhar que, no presente estudo, apenas avaliamos a presença de alterações do sono, tal como o recurso à medicação para regular o sono, não tendo sido analisadas outras formas de regulação, como o consumo de substâncias (e.g., álcool), sendo importante a utilização de uma medida da qualidade do sono estandardizada em estudos futuros, tal como a avaliação de outras formas de regulação do sono.

Os resultados obtidos mostram diferenças significativas de sexo em diversas variáveis estudadas, o que revela a existência de especificidades nos pacientes fumantes do sexo feminino e do sexo masculino. Considerando que as iniciativas de cessação tabágica são apontadas entre as estratégias mais eficazes de melhoria da morbidade associada aos hábitos tabágicos e ainda a necessidade de desenvolver abordagens específicas de acordo com as características do grupo de fumantes que procuram cessar o consumo, mostra-se fundamental o desenvolvimento de programas de intervenção voltados à cessação tabágica que contemplem as especificidades dos fumantes, no sentido de promover uma maior adequabilidade e consequentemente uma maior eficácia da intervenção, traduzindo-se em uma menor recaída dos pacientes. Nesse sentido, e uma vez que as percepções, atitudes e comportamentos de consumo de tabaco diferem entre homens e mulheres (Ferketich, Khan, \& Wewers, 2006), mostra-se fundamental conhecer de forma mais aprofundada esta e outras variáveis (e.g., momento do dia de maior consumo, contexto interpessoal de consumo, pressão para deixar de fumar, sono) em função do sexo, de modo a reunir informação que permitam elaborar programas de intervenção mais ajustados às especificidades e necessidades de sexo dos fumantes portugueses. Em futuras investigações acerca dessa temática, consideramos pertinente estudar a variação dos resultados no sexo feminino e masculino nas seguintes variáveis: encaminhamento para a consulta de cessação tabágica (motivação pessoal, pressão interpessoal ou por indicação do profissional de saúde); idade de início do consumo de cigarros, cronicidade e padrão do consumo; tentativas anteriores de cessação do consumo; sintomas de abstinência; valorização do peso corporal e expectativas em relação ao aumento de peso resultante da cessação do consumo; crenças acerca da eficácia do programa de cessação tabágica; percepção de controle pessoal e autoeficácia para a cessação do consumo; percepção dos benefícios a curto e médio-prazo associados quer ao consumo quer à cessação tabágica; percepção das consequências da cessação tabágica; percepção subjetiva de saúde e de doença; traços de personalidade e psicopatologia; estratégias de coping; percepção subjetiva da pressão social para cessar o consumo; necessidade de contato interpessoal/ suporte social; e intenção de mudança de comportamento tabágico. De fato, a inexistência de resultados relativos à avaliação da motivação para a mudança de comportamento e a não aplicação de escalas de gravidade da dependência e de escalas que permitam a avaliação de psicopatologia constituem limitações do presente estudo.

Sumariamente, as diferenças encontradas entre a amostra feminina e a masculina permitem confirmar a pertinência de atentar às especificidades de sexo no desenvolvimento e implementação das medidas de cessação tabágica. De fato, homens e mulheres fumantes evidenciam especificidades de consumo distintas, quer no que concerne ao padrão de 
consumo, quer em relação ao contexto ambiental e social dos hábitos tabágicos. Assim, e sendo comuns as recidivas verificadas nos fumantes que tentam cessar o consumo, tendendo o insucesso a ser mais elevado nas mulheres, mostra-se primordial investir no desenvolvimento de programas de intervenção ajustados às especificidades de sexo. Para tal, mostra-se fundamental conhecer o contexto social, emocional e ambiental do consumo tabágico, desde os fatores envolvidos no início do mesmo às múltiplas variáveis associadas à manutenção do consumo, procurando considerar sempre à motivação comportamental para a mudança e a fase em que o fumante se encontra no processo de tomada de decisão pela aplicação dos modelos de mudança de comportamento que podem ajudar a explicar o comportamanto tabágico (e.g., Teoria do Comportamento Planeado; Ajzen, 1991; Ajzen \& Madden, 1986; HAPA, Schwarzer, 2008). Poder-se-á, assim, garantir uma maior adequação dos objetivos das medidas de cessação tabágica às necessidades específicas dos fumantes ou das fumantes.

\section{Referências}

Afonso, F., \& Pereira, M. G. (2013). Papel mediador das representações do tabaco e do suporte do parceiro em fumantes e ex-fumantes. Temas em Psicologia, 21, 391-402. doi: 10.9788/TP2013.2-07

Ajzen, I. (1991). The theory of planned behavior. Organizational Behavior and Human Decision Processes, 50, 179-211. doi:10.1016/0749-5978(91)90020-T

Ajzen, I., \& Madden, T. J. (1986). Prediction of goaldirected behavior: Attitudes, intentions, and perceived behavioral control. Journal of Experimental Social Psychology, 2, 453474. doi:10.1016/0022-1031(86)90045-4

Alberg, A. J., Carter C. L., \& Carpenter M. J. (2007). Weight gain as an impediment to cigarete smoking cessation: A lingering problem in need of solutions. Preventive Medicine, 44, 296297. doi: 10.1016/j.ypmed.2007.01.001

Audrain-McGovern, J., \& Benowitz, N. L. (2011). Cigarette smoking, nicotine, and body weight. Clinical Pharmacology \& Therapeutics, 90, 164-168. doi: 10.1038/clpt.2011.105

Balbani, A. P. S., \& Montovani, J. C. (2005). Métodos para abandono do tabagismo e tratamento da dependência da nicotina. Revista Brasileira de Otorrinolaringologia, 71, 820-827. doi: 10.1590/ S0034-72992005000600021

Bauer, T., Göhlmann, S., \& Sinning, M. (2006, August). Gender Differences in Smoking Behavior (IZA Discussion Paper No. 2259). Retrieved from http://seepdf.net/doc/pdf/download/ ftp_iza_org--dp2259.pdf

Bjornson, W., Rand, C., Connett, J. E., Lindgren, P., Nides, M., \& Pope, F. (1995). Gender differences in smoking cessation after 3 years in the lung health study. American Journal of Public Health, 85, 223-230. doi: 10.2105/AJPH.85.2.223

Campos, H. M. L. (2008). Caracterização dos hábitos tabágicos dos pais e dos alunos do $1^{\circ}$ ciclo do ensino básico: implicações para a construção de uma intervenção educativa (Unpublished master's thesis). Instituto de Educação e Psicologia, Universidade do Minho, Portugal.
Catalán, T. V., Gallego-Gómez, J. I., González-Moro, M. T. R., \& Simonelli-Muñoz, A. J. (2014). Fatores influentes na qualidade do sono em pacientes internados. Revista Portuguesa de Enfermagem de Saúde Mental, 12, 43-50. Recuperado de http:// www.scielo.mec.pt/scielo.php?script=sci_arttext\&pid=S164721602014000300006\&lng=pt\&tlng=pt.

Centers for Disease Control and Prevention. (2010). How Tobacco Smoke Causes Disease: The Biology and Behavioral Basis for Smoking-Attributable Disease: A Report of the Surgeon General. Nicotine Addiction: Past and Present. Recuperado de http://www.ncbi.nlm.nih.gov/books/NBK53018/

Chaudhuri, R., Livingston, E., McMahon, A.D., Lafferty, J., Fraser, I., Spears, M., ... Thomson, N. C. (2006). Effects of smoking cessation on lung function and airway inflammation in smokers with asthma. American Journal of Respiratory and Critical Care Medicine, 174, 127-133. doi:10.1164/rccm.200510$15890 \mathrm{OC}$

Christakis, N. A., \& Fowler, J. H. (2008). The collective dynamics of smoking in a large social network. The New England Journal of Medicine, 358, 2249-2258. doi: 10.1056/NEJMsa0706154

Costa, E. C. V., \& Valério, M. J. (2009). Questionário da Consulta de Desabituação Tabágica. Braga: Consulta de Desabituação Tabágica do CHVNG/E.

Direção Geral da Saúde. (2013). Portugal: Prevenção e controlo do tabagismo em números - 2013. Programa Nacional para a Prevenção e Controlo do Tabagismo. Portugal: Ministério da Saúde.

Dollar, K. M., Homish, G. G., Kozlowski, L. T., \& Leonard, K. E. (2009). Spousal and alcohol-related predictors of smoking cessation: A longitudinal study in a community sample of married couples. American Journal of Public Health, 99, 231233. doi: 10.2105/AJPH.2008.140459

Donzé, J., Ruffieux, C., \& Cornuz, J. (2007). Determinants of smoking and cessation in older women. Age and Ageing, 36, 53-57. doi: 10.1093/ageing/afl120

Ferketich, A. K., Khan, Y., \& Wewers, N. E. (2006). Are physicians asking about tobacco use and assisting with cessation? Results from the 2001-2004 national ambulatory medical care survey. Preventive Medicine, 43, 472-476. doi:10.1016/j. ypmed.2006.07.009

Helgeson, V. S. (2016). The psychology of gender. New York: Taylor $\&$ Francis Publishers.

Khaled, S. M., Bulloch, A., Exner, D. V., \& Patten, S. B. (2009). Cigarette smoking, stages of change, and major depression in the Canadian population. La Revue Canadienne de Psychiatrie, 54(3), 204-208.

Leinsalu, M., Tekkel, M., \& Kunst, A. E. (2007). Social determinants of ever initiating smoking differ from those of quitting: A cross-sectional study in Estonia. European Journal of Public Health, 17, 572-578. doi: 10.1093/eurpub/ckm030

Leventhal, A. M., Boyd, S., Moolchan, E. T., Waters, A. J., Lerman, C., \& Pickworth, W. B. (2007). Gender differences in acute tobacco withdrawal: Effects on subjective, cognitive, and physiological measures. Experimental and Clinical Psychopharmacology, 15, 21-36. doi: 10.1037/10641297.15.1.21

Macedo, C. R. (2008). Bruxismo do sono. Revista Dental Press de Ortodontia e Ortopedia Facial, 13, 18-22. doi: 10.1590/ S1415-54192008000200002 
Machado, A. Nicolau, R., \& Dias, C.M. (2009). Consumo de tabaco na população: Análise dos dados do Inquérito Nacional de Saúde 2005/2006. Lisboa: Instituto Nacional de Saúde Doutor Ricardo Jorge - Departamento de Epidemiologia.

May, S. \& Wets, R. (2000). Do social support interventions ("buddy systems") aid smoking cessation? A review. Tobacco Control, 9, 415-422. doi: 10.1136/tc.9.4.415

Nunes, E., Candeias, A., Mendes, B., Pardal, C., Fonseca, J., Oliveira, L., ... Vinagre, S. (2007). Cessação Tabágica. Programa-tipo de actuação em cessação tabágica . Lisboa: Direcção-Geral da Saúde - Gradiva Publicações.

Oliveira, C. M., \& Gorayeb, R. (2012). Diferenças de gênero e fatores motivacionais para início do tabagismo em adolescentes. Saúde \& Transformação Social, 3(1), 49-54. Recuperado de http:// pepsic.bvsalud.org/scielo.php?script=sci_arttext\&pid=S2178$70852012000100008 \& \operatorname{lng}=$ pt\&tlng=pt.

Pereira, C. F., \& Vargas, D. (2015). Profile of women who carried out smoking cessation treatment: A systematic review. Revista de Saúde Pública, 49, 1-8. doi: 10.1590/S00348910.2015049005783

Perkins, K. A., Levine, M., Marcus, M., Shiffman, S., D’Amico, D., Miller, ... Broge, M. (2000). Tobacco withdrawal in women and menstrual cycle phase. Consulting Clinical Psychology, 68(1), 176-180. doi: 10.1037//0022-006X.68.1.176

Prochaska, J. O., DiClemente, C. C., Velicer, W. F., Ginpil, S., \& Norcross, J. C. (1985) Predicting change in smoking status for self-changers. Addict Behaviour, 10, 395-406. doi: 10.1016/0306-4603(85)90036-X

Prosise, G. L., Bonnet, M. H., Berry, R. B., \& Dickel, M. J. (1994). Effects of abstinence from smoking on sleep and daytime sleepiness. Chest, 105, 1136-1141. doi:10.1378/ chest.105.4.1136

Pullon, S., Mcleod, D., Benn, C., Viccars, A., White, S., Cookson, T., ... Green, R. (2003). Smoking cessation in New Zealand: Education and resources for use by midwives for women who smoke during pregnancy. Health Promotion International, 18(4), 315-325.

Ratneswaran, C., Chisnall, B., Drakatos, P., Sivakumar, S., Sivakumar, B., Barrecheguren, ... Steier, J. (2014). A crosssectional survey investigating the desensitisation of graphic health warning labels and their impact on smokers, nonsmokers and patients with COPD in a London cohort. BMJ Open, 4(7): e004782. doi: 10.1136/bmjopen-2013-004782

Rise, J., Kovac, V., Kraft, P., \& Moan, I. S. (2008). Predicting the intention to quit smoking and quitting behaviour: Extending the theory of planned behaviour. British Journal of Health Psychology, 13, 291-310. doi: 10.1348/135910707X187245

Rohrbaugh, M. J., Shoham, V., \& Dempsey, C. L. (2009). Gender differences in quit support by partners health-compromised smokers. Journal of Drug Issues, 39(2), 329-334.

Rosendo, I., Fonseca, G., Guedes, A. R., \& Martins, V. (2009). A characterisation of smokers and factors influencing motivation to stop smoking. Revista Portuguesa de Pneumologia, 15, 783-802. doi: 10.1016/S0873-2159(15)30179-3

Scharf, D. M., Dunbar, M. S., \& Shiffman, S. (2008). Smoking during the night: Prevalence and smoker characteristics. Nicotine \& Tobacco Research, 10, 167-178. doi: $10.1080 / 14622200701767787$
Schwarzer, R. (2008). Modeling health behavior change: How to predict and modify the adoption and maintenance of health behaviors. Applied Psychology, 57, 1-29. doi: 10.1111/j.14640597.2007.00325.x

Solty, H., Crockford, D., White, W. D., \& Currie, S. (2009). Cigarette smoking, nicotine dependence, and motivation for smoking cessation in psychiatric inpatients. The Canadian Journal of Psychiatry, 54(1), 36-45.

Streppel, M. T., Boshuizen, H. C., Ocké, M. C., Kok, F. J., \& Kromhout, D. (2007). Mortality and life expectancy in relation to long-term cigarette, cigar and pipe smoking: The Zutphen Study. Tobacco Control, 16, 107-113. doi: 10.1136/ tc. 2006.017715

Sumner, W., Dunaway, M., \& Dillman, D. G. (1998). Cigarette continuity programs and social support for smoking. Archives of Family Medicine, 7(3), 264-268.

Tonnesen, P., Carrozzi, L., Fagerstro, K. O., Gratziou, C., JimenezRuiz, C., Nardinie, S., ... West, R. (2007). Smoking cessation in patients with respiratory diseases: A high priority, integral component of therapy. European Respiratory Journal, 29, 390 - 417. doi: 10.1183/09031936.00060806

Trigo, M. (2007). Cigarros, tabaco e nicotina: Factores biocomportamentais associados ao tabagismo (Unpublished doctoral dissertation). Faculdade de Psicologia e de Ciências da Educação, Universidade de Lisboa, Portugal.

Walsh, P. M., Carrillo, P., Flores, G., Masuet, C., Morchon, S., \& Ramon, J. M. (2007). Effects of partner smoking status and gender on long-term abstinence rates of patients receiving smoking cessation treatment. Addictive Behaviors, 32, 128136. doi:10.1016/j.addbeh.2006.03.027

World Health Organization. (2009). Report on the global tobacco epidemic. Implementing smoking-free environments. The MPOWER package. Geneva: World Health Organization.

World Health Organization. (2012). Global Report: Mortality Attributable to Tobacco. Geneva: World Health Organization.

Willemse, B. W., Postma, D. S., Timens, W., \& ten Hacken, N. H. (2004). The impact of smoking cessation on respiratory symptoms, lung function, airway hyperresponsiveness and inflammation. European Respiratory Journal, 23, 464-476. doi: 10.1183/09031936.04.00012704

Willemse, B. W., Hacken, N. H., Rutgers, B., Lesman-Leegte, I. G., Postma, D. S., \& Timens, W. (2005). Effect of 1-year smoking cessation on airway inflammation in COPD and asymptomatic smokers. European Respiratory Journal, 26, 835-845. doi: 10.1183/09031936.05.00108904

Williams, R. J., Herzog, T. A., \& Simmons, V. N. (2011). Risk perception and motivation to quit smoking: A partial test of the Health Action Process Approach. Addictive Behaviors, 36, 789-791. doi:10.1016/j.addbeh.2011.03.003

Vidrine, D. J. (2009). Cigarette smoking and HIV/AIDS: Health implications, smoker characteristics and cessation strategies. AIDS Education and Prevention, 21, 3-13. doi: 10.1521/ aeap.2009.21.3_supp.3

Recebido em 02.10.2013

Primeira decisão editorial em 11.05.2016

Versão final em 07.06.2016

Aceito em 19.07.2016 